\title{
Towards Multi-Directional OCT for Speckle Noise Reduction
}

\author{
L. Ramrath ${ }^{1}$, G. Moreno ${ }^{1}$, H. Mueller ${ }^{2}$, T. Bonin ${ }^{2}$, G. Huettmann ${ }^{2}$, \\ and A. Schweikard ${ }^{1}$ \\ ${ }^{1}$ Institute for Robotics and Cognitive Systems, University of Luebeck, Germany \\ ramrath@rob.uni-luebeck.de, \\ ${ }^{2}$ Institute for Biomedical Optics, University of Luebeck, Germany
}

\begin{abstract}
Multi-directional optical coherence tomography (MD-OCT) applies and extends the concept of angular compounding for speckle noise reduction to the area of OCT imaging. OCT images are acquired from a wide range of angles of view. Averaging of the rotated images therefore requires compensation of the parallax which is achieved by simple image registration for image reconstruction. Test measurements of a sample structure in a low and highly scattering environment show that the method improves the signal-to-noise ratio by a factor of 4 and hence reduces speckle noise significantly. Experimental results also show that the proposed averaging increases the performance of common edgedetection algorithms.
\end{abstract}

\section{Introduction}

OCT is an evolving imaging technique with important contributions to several medical applications. Based on the principle of white light interferometry, it presents a high resolution non-invasive imaging technique in certain disciplines e.g. ophthalmology, dermatology, urology, and brain morphology [1]. The most severe problem in OCT imaging for diagnostic purposes is image corruption by speckle noise which complicates further analysis. A wealth of image-based speckle reduction filters have therefore been proposed [2]. All of these approaches, however, feature the disadvantage of altering structural information of the image. Another option of OCT image enhancement is spatial compounding by averaging multiple images by e.g. slightly displacing the sample [3]. These displacements eventually change the speckle patterns and a subsequent averaging process will improve image quality by approximately the square root of the number of averaged images. In this context, the concept of angular compounding by acquiring images from different angles has been proposed [4]. In previous studies, averaging of images was done without applying geometric image transformations. Thus, the effect of parallax was not compensated for which falsifies the averaged image. Also, in case of small displacements, speckle pattern might not really be independent and averaging is not suitable.

In this work, the concept of angular compounding is augmented by an automated parallax compensation applying image registration methods to construct

D. Metaxas et al. (Eds.): MICCAI 2008, Part I, LNCS 5241, pp. 815-823, 2008.

(C) Springer-Verlag Berlin Heidelberg 2008 


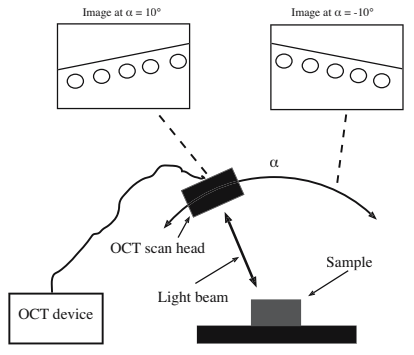

Fig. 1. The concept of multidirectional OCT

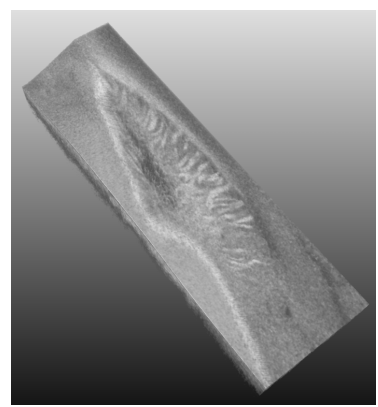

Fig. 2. Three-dimensional reconstruction of OCT B-scans of the rat brain showing the striatum with embedded white fibres

an averaged image. For that reason, an angular scanning method is proposed which is compatible with commercial OCT instruments. Applying the mentioned approach enhances structural image properties while reducing speckle noise contribution. An exemplary field of application include the neurosurgical scenario. Here, a robot-assisted microscope with an integrated OCT system is used to acquire multi-directional images automatically [5]. Such a system is able to provide the surgeon with information at microscale level leading to e.g. robust identification of residual tumor tissue or white matter fibres (see Fig. 2).

\section{Method}

\subsection{Concept and Background of Multi-Directional OCT}

The occurence of speckle noise is an inherent problem of OCT as it uses the coherence of backreflected light. An important characteristic of speckle is the statistical nature of the speckle pattern. If the scanning setup is not subject to any changes (e.g. movement, temperature), the speckle pattern is time-invariant. It is, however, spatially variant and scanning a sample from different directions will consequently yield a different speckle pattern. Speckle noise is usually modeled as a random multiplicative noise [1]. If $f$ denotes the ideal image and $g$ the speckled image, this assumption can be expressed as

$$
g=f u,
$$

where $u$ is a signal-independent random variable. Appyling the logarithmic operator to Eq. 1 transforms the multiplicative noise into a additive noise. In [6], the authors show that the additive noise component is approximately Gaussian distributed noise. This, in turn, motivates the use of a simple mean operator to estimate the structural component $f$. As the speckle pattern $u$ is time-invariant 
averaging can be performed by taking B-scans from multiple direction. Averaging directional images thus reduces the Gaussian distributed noise but keep the structural information.

The idea of multi-directional OCT is visualized in Fig. 1, The OCT scanning head rotates along a circular path around the sample. For each directional B-scan, the angle of view $\Delta \alpha$ is known which will be of advantage in extracting structural information from speckled images. Images from different directions, however, can not be simply superimposed geometrically as the center of rotation of the scanning head, i.e. the distance to the probe, is not known exactly. Adequate averaging can therefore only be done if the effect of parallax is compensated.

\subsection{Testbed Setup}

To test the performance of the proposed approach, the following testbed scenario is chosen. Instead of rotating the OCT scanning head about the sample, the sample is rotated under the scanning head. The sample is attached to a micromanipulator stage comprising three cartesian stages and one rotary stage (see Fig. 3). The structural element of the sample consists of nylon wires which are chucked parallely with a distance of approximately $500 \mu \mathrm{m}$. This element is placed into a sealed case which features a glas plate on top (see Fig. 44). Sealing is provided in order to put the sample into different media featuring different scattering properties. The mechanical setup is designed such that repetitive measurements of the same sample can be conducted although the scattering medium is changed. The scanning direction is adjusted to run across the wires such that B-scans image the circular cross-section of the wires. Fig. 5 shows a B-scan for an angle of $\alpha=-10^{\circ}$ while Fig. 6 provides an image of the same sample at

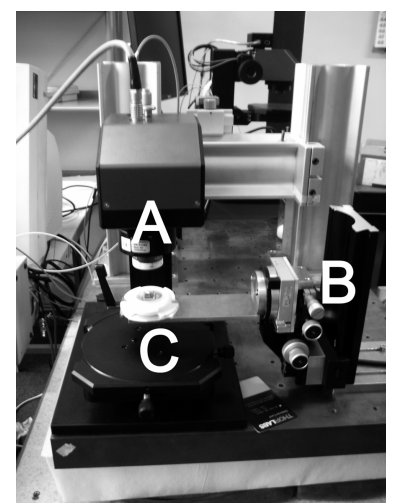

Fig. 3. Scanning setup for multidirectional OCT: [A] scanning head [B] micromanipulator stage for translational and rotational adjustments $[\mathrm{C}]$ sample

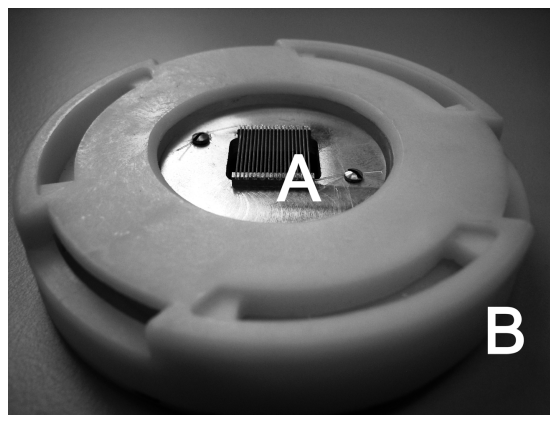

Fig. 4. Sample for multi-directional OCT: [A] nylon wires representing the structural element of the sample [B] sealing case 


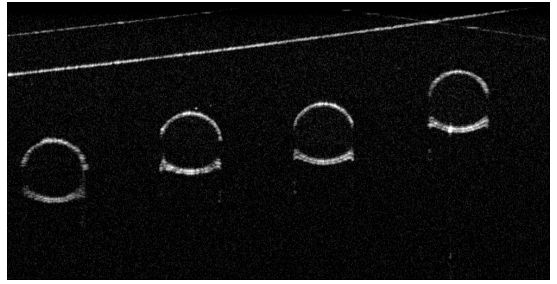

Fig. 5. B-scan showing the crosssections of nylon wires in the aqueous environment at $\alpha=-10^{\circ}$. The white line in the upper part corresponds to the sealing glas plate.

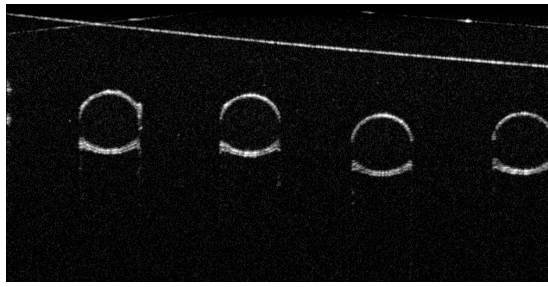

Fig. 6. B-scan showing the crosssections of nylon wires in the aqueous environment at $\alpha=10^{\circ}$. The white line in the upper part corresponds to the sealing glas plate.

$\alpha=10^{\circ}$. Two imaging sequences with a stepsize of $\Delta \alpha=1^{\circ}$ are obtained, both having an angular range of $\alpha=\left[-10^{\circ} \ldots 10^{\circ}\right]$. For the first scenario, an aqueous environment was chosen which corresponds to a low scattering environment hereafter called water images. The second imaging sequence was done with the nylon wires embedded into a highly scattering milk suspension (10\% milk, 90\% water) which will be referred to as milk images.

\subsection{Automated Registration Technique for Parallax Compensation}

Imaging from different directions introduces the effect of parallax and establishes the need of an image correction for adequate averaging. This work proposes an automated registration method especially adapted to the described setup to provide the proof of concept of MD-OCT. This does, however, not limit the generality of the proposed approach as in other applications, other registration methods can be applied (see e.g. [7]).

As the sample geometry is not changed in the MD-OCT scenario, registration in the following is performed as a rigid registration consisting of translation and rotation [7. The reference image is defined to be the zero degree image. As the angle of rotation of each image is known, the first step is to back-rotate all images to approximate the reference's angle. The back-rotation algorithm considers an arbitratry point outside the image as the center of rotation which does usually not correspond to the center of rotation of the scanning head. The back-rotated image therefore requires an additional alignement for correct averaging. Determination of the alignment parameters is based on a characteristic property of the obtained cross-sectional images. The single fibers from the sample appear to be circles with well-defined borders well suited for template matching. Analyzing the correlation with circular templates provides the coordinates of the circle centers. Additionally, a field of view is defined to delete the edges of the images that the whole set doesn't share. Knowing the location of the circles in the back-rotated and the reference image, the translational part of the registration is determined. 


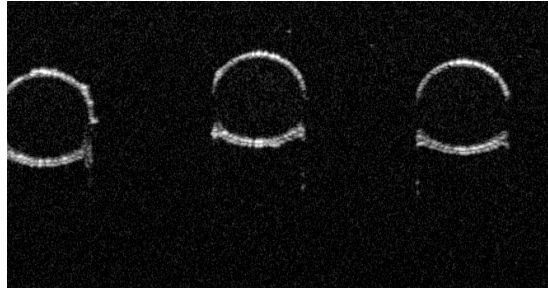

Fig. 7. B-scan showing the crosssections of nylon wires in the aqueous environment at $\alpha=0^{\circ}$

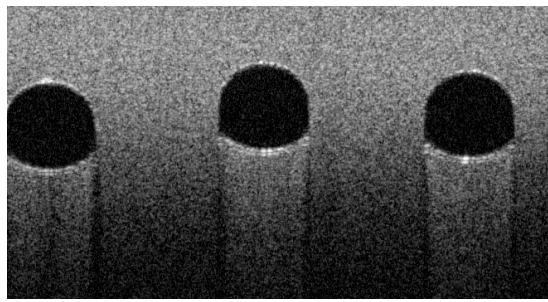

Fig. 9. B-scan showing the crosssections of nylon wires in the milk suspension environment at $\alpha=0^{\circ}$

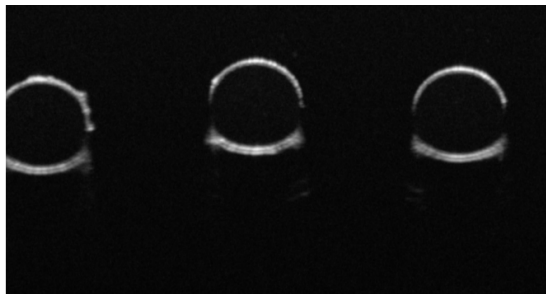

Fig. 8. Averaged of 21 B-scan from different angles showing the cross-sections of nylon wires in the aqueous environment

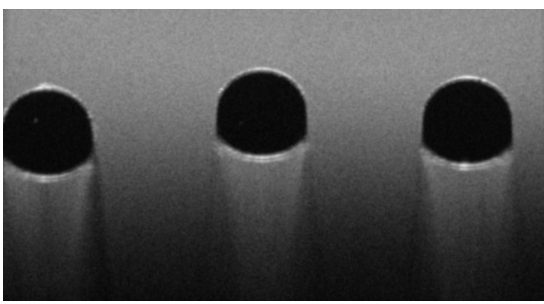

Fig. 10. Averaged of 21 B-scan from different angles showing the crosssections of nylon wires in the milk suspension environment

\section{Results}

Fig. 7 shows a B-scan of the sample for $\alpha=0^{\circ}$. The average of all B-scans over an angular range $\alpha=\left[-10^{\circ} \ldots 10^{\circ}\right]$ after registration is visualized in Fig. 8 Analogous, results for the milk environment are given in Fig. 9 (showing a B-scan from a single angle of view) and Fig. 10 (showing the averaged results after registration). To evaluate the performance of the proposed approach, different measures were analyzed. First, the signal-to-noise ratio was evaluated for an increasing number of viewing directions. The SNR is evaluated as the ratio of the mean and the standard deviation. Fig. 11] shows the SNR course for an increasing number of B-scans acquired from different angular directions for the aqueous test scenario. For the milk trials, results are given in Fig. 12, It can be seen that the SNR improves by almost a factor of 4 for water and milk. An additional performance evaluation is based on the capability of structure identification which is of great importance in medical image processing. In the following, identification refers to the detection of the circular borders in different scattering environments. Although no true OCT image of the sample in our test scenario is known, an approximation can be made by assuming the averaged water result as the ground truth. This assumption is supported by the low 


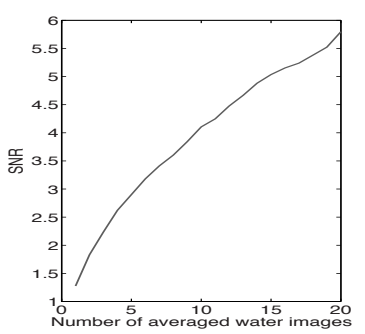

Fig. 11. SNR for an increasing number of averaged water images

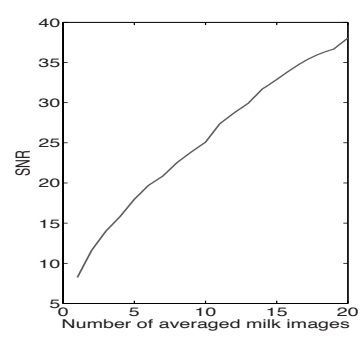

Fig. 12. SNR for an increasing number of averaged milk images

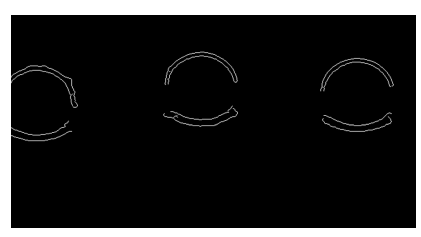

Fig. 13. Edge detection with the Canny filter in averaged water image (ground truth)

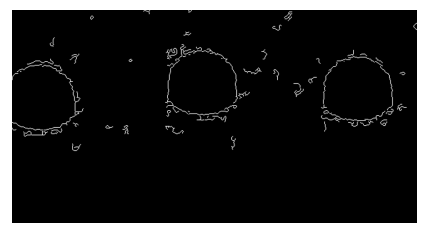

Fig. 14. Edge detection with the Canny filter in single view milk image

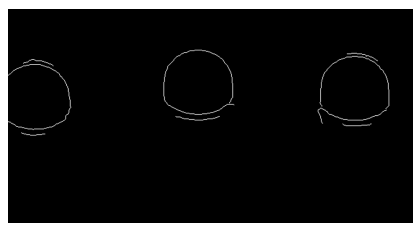

Fig. 15. Edge detection with the Canny filter in averaged milk image

scattering properties of water and the high SNR of the averaged B-scan (see Fig. 11). Equal geometrical settings of the measurement scenarios for both tests allow a direct comparison of structures identified in the ground truth (averaged water image) and images taken from the milk suspension testing. Edge detection is now performed by using the Canny operator. All following edge detection operations were performed with constant settings, no parameter adjustments have been made for different images. Fig 13 shows the edges detected in the average water image, Fig 14 the edges detected in $\alpha=0^{\circ}$ in milk suspension, and Fig. 15 the results of the edge detection on the averaged milk suspension. Clearly, the best edge detection is provided for the averaged water image. While edges in the single view milk images are irregular and non-existing edges are detected, the edge detection for the averaged milk images is much more similar to the averaged water one. To support these findings numerically, the correlation coefficient of the edge images of the averaged water B-scan and the averaged milk suspension images is given in Fig. 16. Additionally, the mean-square error of between the edge images of the milk enviroment and the averaged water image is plotted 


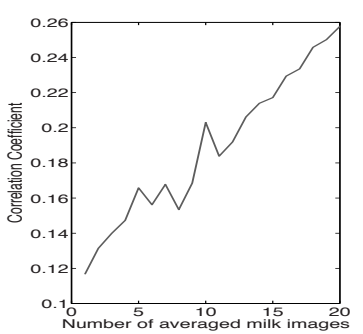

Fig. 16. Correlation performance for edge images with an increasing number of of averaged milk images and the averaged water (ground truth) image

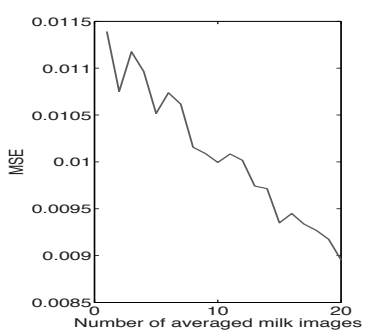

Fig. 17. MSE performance for edge images with an increasing number of averaged milk images and the averaged water (ground truth) image

in Fig. 17. To compare the structure enhancement to existing approaches, edge detection performance on filtered images is analyzed. Filter performance of common filters as the median filter, the Frost filter, and the Perona-Malik filter are provided in Tab. 1 .

\section{Discussion}

From visual inspection of the results shown in Fig. 8 and Fig. 10, it can be seen that the effect of speckle noise is clearly reduced while the structural elements, namely the wires, are enhanced. The reduction of speckle noise is also quantitatively supported by the course of the SNR which increases almost by the factor 4. This corresponds to theory which states that averaging improves the SNR by $\sqrt{n}=\sqrt{21} \approx 4.5$ where $n$ is the number of images averaged. Note that the larger part of the improvement is achieved by averaging 10 images, while the remaining 10 images only provide a smaller improvement. As multi-directional imaging takes effort, e.g time consumption, the user has to decide which image quality serves his needs best. Note that Fig. 9] shows parallel structures (shadows) extending from the bottom of the nylon wires to the bottom of the image. In Fig. 10. these structures are tapered. This effect is related to the experimental setup where the probe is rotated rather than the sample. Shadows will therefore always appear in axial (scanning) direction. If the scanning head is rotated, the shadow direction will change from image to image thus reducing the tapering. Structure identification is also improved with the proposed technique. Simple edge dete-

Table 1. Mean square error between edge images and the averaged water (ground truth) image. Edge images were obtained by applying a simple edge detection after median filtering, Perona-Malik filtering, Frost filter filtering, and the proposed MDOCT approach.

\begin{tabular}{|c|c|c|c|}
\hline Median & Perona-M & Frost & ID-OCT \\
\hline 0.11 & 0.09 & 0.11 & 0.08 \\
\hline
\end{tabular}


cion in averaged images taken from a highly scattering enviroment compares significantly better to edge detection in images in a low scattering environment. This is clearly validated by the increasing correlation coefficients (Fig. 16) and the decreasing MSE (Fig. 17). Compared to existing image filters for speckle noise reduction, the proposed approach performs better than existing filtering techniques (see Tab. 1). Performance of the MD-OCT approach is better than median and Frost filtering while only slightly better than Perona-Malik filtering. The good performance of the Perona-Malik filter in this case, however, might be related to the simple problem and is eventually worse for more complicated structures.

Although registration in the proposed approach is unique to the application, it provides a good proof of concept and indicates the generality of the approach. In clinical applications, especially for in vivo imaging, other methods of registration from a wealth of medical image registration methods can be taken [7]. The experimental testbed setup, however, has clear disadvantages since it is clearly unsuitable for use in-vivo and angular scanning is very slow. Future developments therefore concentrate on improving the hardware setup. An exemplary application includes a motorized OCT-equipped operating microscope for neurosurgery [5] which is able to perform automated angular scannings.

\section{Conclusion}

The concept of multi-directional OCT for speckle noise reduction and structure enhancement has been proposed. It is based on extending the idea of spatial compounding of multi-directional OCT images by a parallax compensation. Sample imaging of certain structures in a low and highly scattering environment show that the method significantly reduces speckle noise in OCT images and simplifies structure identification. The assets of the proposed approach present a valuable advantage for OCT-based medical imaging and subsequent diagnosis. In total, the findings motivate the design of (robot-assisted) OCT systems being able to acquire multi-directional images and thus enabling a system-based speckle noise reduction.

\section{References}

1. Bouma, B.E., Tearney, G.J.: Handbook of Optical Coherence Tomography. Marcel Dekker, Inc., New York (2002)

2. Brezinski, M.E.: Optical Coherence Tomography - Principles and Application. Academic Press, London (2006)

3. Schmitt, J.M., Xiang, S.H., Yung, K.M.: Speckle in optical coherence tomography. Journal of Biomedical Optics 4, 95-105 (1999)

4. Desjardins, A.E., Vakoc, B.J., Oh, W.Y., Motaghiannezam, S.M., Tearney, G.J., Bouma, B.E.: Angle-resolved optical coherence tomography with sequential angular selectivity for speckle reduction. Optics Express 15, 6200-6209 (2007) 
5. Lankenau, E., Klinger, D., Winter, C., Malik, A., Mueller, H., Oelckers, S., Pau, H.-P., Just, T., Huettmann, G.: Combining Optical Coherence Tomography (OCT) with an Operating Microscope. Springer, Heidelberg (2007)

6. Arsenault, H.H., April, G.: Properties of speckle integrated with a finite aperture and logarithmically transformed. J. Opt. Soc. Am. 66, 1160-1163 (1976)

7. Modersitzki, J.: Numerical Methods for Image Registration. Numerical Mathematics and Scientific Computation, Oxford University Press Series (2004) 